\title{
Herausforderungen im internationalen Schienenfernverkehr in Europa
}

\author{
Marco Kampp
}

\section{Entwicklung des internationalen Fernverkehrs in Europa}

Ein wichtiger Bestandteil der grenzüberschreitenden Mobilität in Europa ist der internationalen Schienenfernverkehr. Dieser erlaubt einen umfassenden Zugang zu schneller, sicherer und komfortabler Mobilität für private und berufliche Zwecke und trägt erheblich zur Verwirklichung eines einheitlichen Europas bei.

Dieser Beitrag gibt einen kurzen Überblick über die Entwicklung des internationalen Schienenfernverkehrs und möchte auf bestehende Herausforderungen in einem europäischen Kontext hinweisen.

Die Strecken für den Hochgeschwindigkeitsverkehr in Europa wurden sowohl durch die Mitgliedstaaten der EU als auch die Kommission der Europäischen Union selbst seit vielen Jahren stetig weiterentwickelt. Hochgeschwindigkeitsverkehr (HGV) bezeichnet im allgemeinen Zugverkehr, welcher mit Geschwindigkeiten von mehr als $200 \mathrm{~km} / \mathrm{h}$ durchgeführt werden kann. Einzelne Strecken in Europa können dabei auch durch Züge mit Geschwindigkeiten von bis zu $360 \mathrm{~km} / \mathrm{h}$ bedient werden. Besonders zu nennen sind hier die Strecken zwischen Rom und Mailand in Italien - die „Direttissima“ gilt seit der Inbetriebnahme im Jahre 1977 allgemein als die erste HGV-Strecke in Europa - sowie die Strecken in Frankreich, welche Paris sternförmig mit den wichtigsten Städten des Landes verbinden. Den wirklichen „Startschuss“ in Europa erhielt der HGV durch die Ölkrise in den 1970er-Jahren,

Article note: Marco Kampp ist Leiter Internationaler Fernverkehr der DB Fernverkehr AG. Der Verfasser bringt in diesem Beitrag allein seine persönliche Auffassung zum Ausdruck.

M. Kampp (西)

Leiter internationaler Fernverkehr, DB Fernverkehr AG, Frankfurt am Main, Deutschland

E-Mail: Marco.Kampp@deutschebahn.com

S. Laimer, C. Perathoner (Hrsg.), Mobilitäts- und Transportrecht in Europa,

Bibliothek des Wirtschaftsrechts 2,

https://doi.org/10.1007/978-3-662-63635-0_12 
als viele Länder in Europa bedingt durch die bisherige Abhängigkeit fossiler Brennstoffe nach einer zukunftsfähigen und unabhängigen Alternative suchten.

Diese zunächst strategische Zielstellung zusammen mit dem Ziel einer grenzenlosen Mobilität in Europa sind die Gründe, warum der internationale Schienenverkehr für Personen und Güter ein elementarer Bestandteil der Programmatik der Europäischen Union bilden. Im Hochgeschwindigkeitsverkehr allein hat die EU von 2000-2018 einen Betrag in Höhe von 23,7 Mrd. EUR für die Mitfinanzierung von Infrastrukturvorhaben im Hochgeschwindigkeitsverkehr zu Verfügung gestellt. ${ }^{1}$ Diese wirtschaftliche Förderung zusammen mit den Initiativen der einzelnen Mitgliedstaaten ist deutlich in der Entwicklung und Ausweitung der Hochgeschwindigkeitsinfrastruktur in Europa erkennbar (Abb. 1).

Dieser überaus positiven stetigen Ausweitung der Infrastruktur für den internationalen Fernverkehr in Europa stehen jedoch leider bis heute unterschiedliche Normen und rechtliche Regelungen der einzelnen Mitgliedsstaaten gegenüber, welche den täglichen Betrieb von internationalen Schienenverkehren erschweren und eine sprunghafte Ausweitung des Angebots wie in anderen Sektoren sehr einschränken.

So weisen aus Sicht des Autors die Entwicklung der Marktliberalisierung und die der technischen Standardisierung in Europa unterschiedliche Geschwindigkeiten in Europa auf. Der grenzüberschreitende Verkehr muss sich daher noch heute mit Eigenarten auseinandersetzen, die im Zeitalter der Digitalisierung geradezu anachronistisch anmuten.

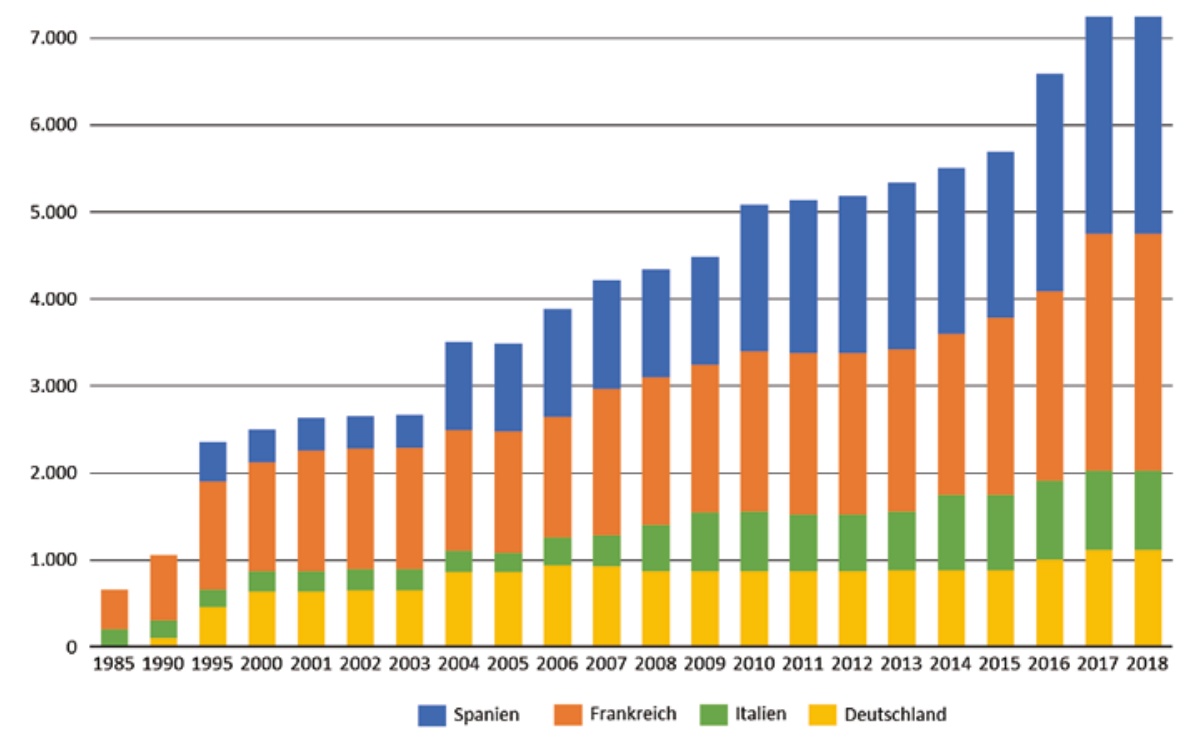

Abb. 1 Länge der Hochgeschwindigkeitsschienennetze in der EU (eigene Darstellung) - Wachstum von 1985-2017. (Quelle: Internationaler Eisenbahnverband UIC)

\footnotetext{
${ }^{1}$ Ein europäisches Hochgeschwindigkeitsnetz (19/2018), Amt für Veröffentlichung der Europäischen Union, ISBN 978-92-847-0062-2.
} 


\section{Der internationale Fernverkehr der Deutschen Bahn AG}

Die Deutsche Bahn AG ist einer der größte Mobilitäts- und Logistikunternehmen weltweit. Wesentliches Element der Strategie „Starke Schiene“ ist das Ziel, die Vernetzung von Menschen und Gütern in Europa nachhaltig zu verbessern und einen Beitrag ,für Europa“ zu leisten. Neben der Weiterentwicklung des grenzüberschreitenden Güter- und Regionalverkehrs kommt dem internationalen Fernverkehr hier ein zentraler Stellenwert in der Umsetzung der Strategie bei.

Der internationale Fernverkehr der DB bedient bereits heute erfolgreich ein dichtes Streckennetz zwischen deutschen sowie europäischen Metropolen. Über 150 Ziele in den Nachbarländern Deutschlands sowie zusätzlich in Italien, der Slowakei, Ungarn und Kroatien sind mit dem Fernverkehr von Deutschland aus erreichbar. In Summe befördern mehr als 240 internationale Fernverkehrszüge über 40.000 Fahrgäste pro Tag. Der Umsatz mit grenzüberschreitenden Verkehren im Tagesfernverkehr konnte seit 2009 durch Angebotsausweitungen in Verbindung mit dem starken Zuwachs der Nachfrage um mehr als $60 \%$ wachsen (vgl. Abb. 2).

Die Mehrzahl der Verbindungen ins europäische Ausland werden in Kooperation zwischen DB Fernverkehr sowie ausländischen Partnerunternehmen erbracht. Eigenwirtschaftlich im Wettbewerb agiert DB Fernverkehr dem gegenüber in Belgien auf der Strecke Frankfurt-Köln-Brüssel sowie in Italien auf der Strecke München-Innsbruck-Verona/Bologna/Venedig.

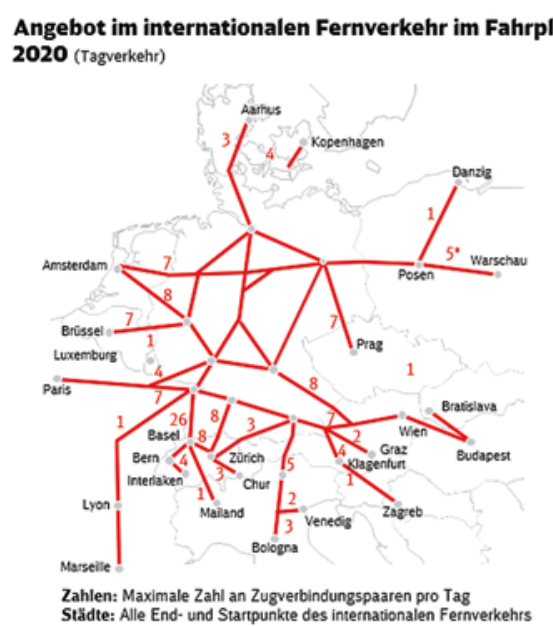

Internationaler Umsatz in den letzten zehn
Jahren um mehr als $\mathbf{6 0 \%}$ gewachsen
Zahlen: Grenzüberschreitender Umsatz im Tagesfernverkehr in Mio. EUR

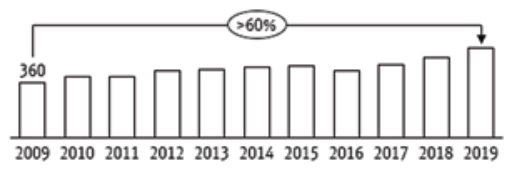
Grenzüberschreitenden Verkehre werden in
Kooperation mit Partnern erbracht

Abb. 2 Angebot im internationalen Fernverkehr der Deutschen Bahn AG und Umsatzwachstum (eigene Darstellung). (Quelle: DB Fernverkehr AG) 


\section{Herausforderungen und Ansätze zur Weiterentwicklung}

Die bereits in der Einleitung angedeuteten Herausforderungen im internationalen Fernverkehr stellen die Eisenbahnunternehmen in Europa jeden Tag aufs Neue auf die Probe. Grundvoraussetzung für ein internationales Angebot ist eine Infrastruktur, die nicht an der Grenze der Mitgliedsstaaten in Europa endet, sondern durchgehend gleiche Kapazität und Geschwindigkeit ermöglicht. Für einen durchgängigen Betrieb sind zusätzlich zur Eisenbahninfrastruktur Züge notwendig, die in verschiedenen Ländern fahren können und die dortigen entsprechenden Voraussetzungen erfüllen.

Der Auf- und Ausbau von Infrastruktur ist eine der wesentlichen Aufgaben des Staates. Im Konflikt zwischen europäischen und nationalen Interessen besteht die Herausforderung, einen Kompromiss zwischen diesen zu finden. Oftmals leiden dabei die Interessen der grenzüberschreitenden Verkehre und einheitliche Regelungen in Europa existieren nicht (Abb. 3).

Exemplarisch aber nicht vollständig sei hier aufgeführt, dass

- Grenzübergänge sich heute in der Regel im Eisenbahnverkehr für Reisende noch deutlich spürbar gestalten und beispielsweise die Reisegeschwindigkeit erheblich eingeschränkt ist.

- Vielmals Halte am Grenzpunkt notwendig sind, da andere technische Regeln Anwendung finden müssen und die Personale aufgrund unterschiedlicher Rechtsnormen wechseln müssen.

- Es technisch keinen einheitlichen Standard in Europa gibt - es existieren in Europa 4 verschiedene Stromsysteme, jedes Land besitzt eigene Signalsystem (bis zu 20 verschiedene Systeme in Europa) und Zulassungsvoraussetzungen für Fahrzeuge und die Qualifizierung von Personal sind nicht europaweit geregelt.

Damit internationale Verbindungen durchgehend angeboten werden können, müssen operativ alle Anforderungen erfüllt werden. Dies stellt die Betreiber von internationalen Verkehren vor große Herausforderungen und ist in vielen Fällen technisch und organisatorisch lösbar. Die Kosten für den internationalen Fernverkehr sind dadurch jedoch deutlich höher als im rein nationalen Verkehr oder im intermodalen Wettbewerb (Flugverkehr, Fernbus, PKW). Dem internationalen Fernverkehr entstehen dadurch wirtschaftlich erhebliche Nachteile im Vergleich zu anderen Verkehrsträgern. Internationale Kooperationen erhalten dadurch eine besondere Bedeutung, da zusätzliche Kosten gemeinsam getragen werden können. Darüber hinaus erzielt der internationale Fernverkehr durchschnittlich geringere Erträge, da über die langen Entfernungen die Durchschnittpreise in der Regel sinken. Die Erfolgsrechnung für internationale Fernverkehre ist daher anspruchsvoll und zeichnet sich durch geringe Margen aus.

Zur Verdeutlichung der unterschiedlichen Anforderungen in Europa sei auf das Thema der Zulassung hingewiesen. Beispielsweise besitzt die seit vielen Jahren in Europa eingesetzte Lokomotive Taurus 1216 (Siemens ES64U4) der ÖBB bis heute den Geschwindigkeitsrekord für Lokomotiven mit einer nachgewiesenen 


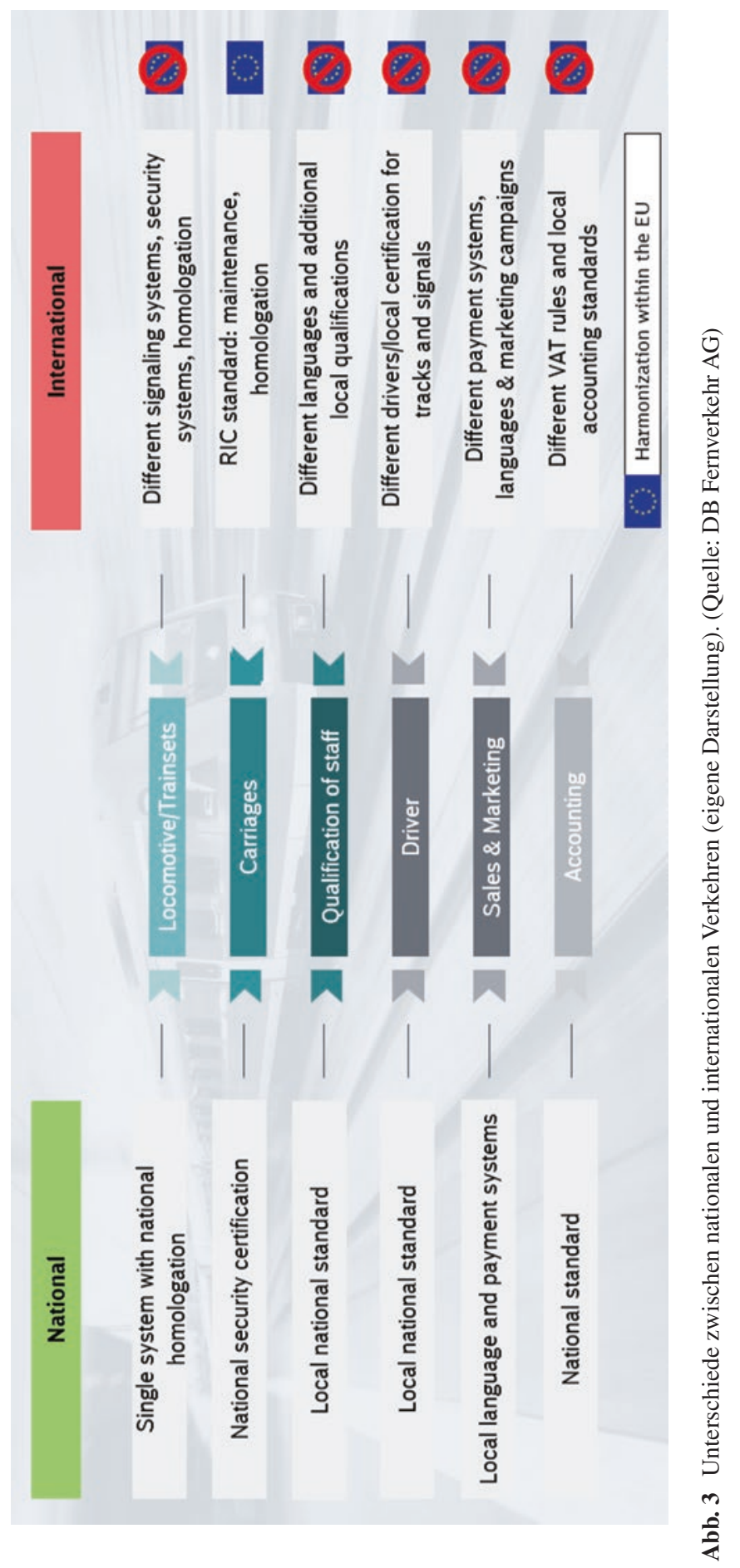


Höchstgeschwindigkeit von $357 \mathrm{~km} / \mathrm{h}$. Die Zulassung für den Betrieb von Personenverkehr mit einer Reisegeschwindigkeit von $230 \mathrm{~km} / \mathrm{h}$ liegt in vielen Ländern Europas vor, für Italien, Slowenien und Tschechien sind die Lokomotiven bis heute jedoch lediglich für $160 \mathrm{~km} / \mathrm{h}_{\text {zugelassen. }}{ }^{2}$

Als zweites Beispiel lässt sich das Zugsicherungssystem ETCS anführen, welches als European Train Control System geplant wurde, um genau die bestehenden Einschränkungen der nationalstaatlichen Zugsicherungssysteme zu überwinden. Heute gibt es jedoch viele unterschiedliche und inkompatible Untervarianten, einen nur eingeschränkten Wettbewerb auf Seiten der Hersteller der Bahnindustrie und zum Teil spezifische Ausprägungen je Land. ${ }^{3}$

Die mangelnde Standardisierung ist ein wichtiger Grund, warum ein grenzüberschreitender Anbieter im Schienenpersonenfernverkehr im Vergleich zu Flugindustrie erhebliche wirtschaftliche Kostennachteile in Kauf nehmen muss. Die Voraussetzungen und Rahmenbedingungen für Wettbewerb, wie sie in anderen Netzwerkindustrien wie beispielsweise im Bereich Telekommunikation oder Flugverkehr üblich sind, erfordern neben einer Marktliberalisierung und dem uneingeschränkten Zugang zu diesem zwingend einen hohen Grad an Standardisierung.

Flugzeuge können beispielsweise überall auf der Welt das gleiche Kerosin tanken. Ersatzzeile können weltweit gewartet und ersetzt werden. Eine Zulassung erfolgt ebenfalls weltweit und Piloten brauchen eine typspezifische Ausbildung und Qualifizierung, können sonst aber in der Regel an allen großen Flughäfen der Welt starten und landen. Die Flugsprache ist einheitlich Englisch.

\section{Ausblick}

Der internationale Zugverkehr stellt nicht nur in Zeiten wachsender klimapolitischer Herausforderungen die beste ökologische Reisevariante da. Mit dem 4. Eisenbahnpaket wurden durch die Europäische Union bereits vielfältige Ansätze zur Weiterentwicklung und weiteren Standardisierung in Europa geschaffen. ${ }^{4} \mathrm{Um}$ zukunftsfähig in Europa klimafreundliche Mobilität anbieten zu können gilt es daher, politisch und gesetzgeberisch den internationalen Verkehr soweit zu berücksichtigen, dass faire Rahmenbedingungen $\mathrm{zu}$ anderen Verkehrsträgern hergestellt werden. Konkret kann dies durch Initiativen umgesetzt werden, welche sich entlang folgender Leitplanken orientieren:

1. Angleichung intermodaler Wettbewerbsbedingungen für den internationalen Zugverkehr. Dies kann zum Beispiel durch die Aufhebung der Mehrwertsteuer auf internationale Zugfahrkarten, die Reduzierung der Trassenpreise für internationale Verbindungen sowie eine Gleichbehandlung bei der Energiebesteuerung erreicht werden.

\footnotetext{
${ }^{2}$ https://de.wikipedia.org/wiki/Siemens_ES64U4.

${ }^{3}$ https://de.wikipedia.org/wiki/European_Train_Control_System.

${ }^{4}$ https://de.wikipedia.org/wiki/Viertes_Eisenbahnpaket.
} 
2. Einrichtung einer Europäischen Investitionsförderung für international einsetzbares Rollmaterial im Rahmen des Green Deal. Fokussierung der EU auf die Leistungsfähigkeit der ERA und länderunabhängige Standards bei der Neugestaltung europaweiter Zulassungen.

3. Einführung einer expliziten wettbewerbsrechtlichen Ausnahme für Kooperationen im internationalen Bahnverkehr zur Förderung grenzüberschreitender Verbindungen.

Aufgrund der unterschiedlichen internationalen Standards und der langen Zyklen der Anpassung sind Kooperationen für ein attraktives Angebot im internationalen Verkehr weiterhin unerlässlich und perspektivisch die Entwicklung durchgehender Standards entscheidend.

Open Access Dieses Kapitel wird unter der Creative Commons Namensnennung 4.0 International Lizenz (http://creativecommons.org/licenses/by/4.0/deed.de) veröffentlicht, welche die Nutzung, Vervielfältigung, Bearbeitung, Verbreitung und Wiedergabe in jeglichem Medium und Format erlaubt, sofern Sie den/die ursprünglichen Autor(en) und die Quelle ordnungsgemäß nennen, einen Link zur Creative Commons Lizenz beifügen und angeben, ob Änderungen vorgenommen wurden.

Die in diesem Kapitel enthaltenen Bilder und sonstiges Drittmaterial unterliegen ebenfalls der genannten Creative Commons Lizenz, sofern sich aus der Abbildungslegende nichts anderes ergibt. Sofern das betreffende Material nicht unter der genannten Creative Commons Lizenz steht und die betreffende Handlung nicht nach gesetzlichen Vorschriften erlaubt ist, ist für die oben aufgeführten Weiterverwendungen des Materials die Einwilligung des jeweiligen Rechteinhabers einzuholen. 